\title{
25 Research Square \\ Effects of Nighttime Aromatherapy using Skin Patches with Lavender Aroma on Mood States in Young Women
}

\section{Kanako Sazawa}

Teikyo University - Hachioji Campus: Teikyo Daigaku - Hachioji Campus

Yoshiko Ogawa ( $\nabla$ yogawa@main.teikyo-u.ac.jp)

Teikyo University - Hachioji Campus: Teikyo Daigaku - Hachioji Campus https://orcid.org/0000-00019083-5586

\section{Shigeo Kawada}

Teikyo University - Hachioji Campus: Teikyo Daigaku - Hachioji Campus

\section{Research}

Keywords: aromatherapy, lavender, skin patch, mood, stress

Posted Date: April 26th, 2021

DOI: https://doi.org/10.21203/rs.3.rs-417240/v1

License: (c) (i) This work is licensed under a Creative Commons Attribution 4.0 International License. Read Full License 


\section{Abstract}

Background: The effect of nighttime aromatherapy on sleep has been explored. However, its effect on mood states has not been established. This study aimed to determine the effects of nighttime aromatherapy, using skin patches with lavender aroma, on mood states of young women.

Methods: Twenty-four young women slept as usual with a skin patch placed under their clothing for seven consecutive nights. One group of subjects (AROM group) used skin patches with lavender aroma, whereas the other group (CONT group) used skin patches without the aroma. The primary outcome measure was mood states assessed with the Profile of Mood States 2nd Edition (POMS 2). The secondary outcome measures were physiological indices of stress assessed by salivary a-amylase activity, cortisol concentration, and pulse rate measured immediately after waking up. Assessments were performed pre-intervention, the morning after the first intervention night, and post-intervention.

Results: Skin patches with lavender aroma improved total mood disturbance and two negative subscales scores of the POMS 2 in the AROM group over time. There was an interaction between time and treatment condition (with/without aromatherapy) for the fatigue-inertia subscale, although no interaction was detected between time and condition for total mood disturbance and the anger-hostility subscale. The positive subscales scores of POMS 2 did not change in both groups. In the AROM group, salivary aamylase level measured the morning after the first intervention night was significantly higher than the pre-intervention level; however, there was no significant difference between pre- and post-intervention. Moreover, there were no significant changes in salivary cortisol concentration and pulse rate in both groups.

Conclusions: Unlike the AROM group, no improvement in mood states was observed in the CONT group. Low-cost and easily applicable aromatherapy using skin patches are potentially useful in stabilizing mood states in various populations.

Trial registration: UMIN-CTR, UMIN000035051. Registered 28 November 2018, https://upload.umin.ac.jp/cgi-open-bin/ctr_e/ctr_view.cgi?recptno=R000039962

\section{Introduction}

Aromatherapy, which uses essential oils from plants, is a representative alternative and complementary therapy. Aromatherapy is widely administered to improve physical, emotional, and spiritual states in healthy persons and in patients with various diseases [1, 2]. In particular, aromatherapy has been demonstrated to be effective for treating pain in dysmenorrhea and labor/childbirth, high blood pressure in hypertension; stress, depression, and sleep problems in hemodialysis patients; stress in healthy adults; anxiety in perioperative patients; and for improving sleep quality in various populations [1].

The Inhalation of essential oils is the most common method of aromatherapy, although the method of application varies widely [1]. The effects of aromatherapy are theorized to result from the binding of the 
chemical components of the essential oil to receptors in the olfactory bulb, impacting the limbic system, which is the brain's emotional center [2]. Lavender, which is one of the most popular essential oils, has effects, such as anxiolytic, mood stabilizing, sedative, relaxing, and antidepressive effects, on the physiological and psychological states of an individual [3-5]. Additionally, lavender aroma has been reported to have positive effects on sleep [6, 7]. Linalool and linalyl acetate, which are the chemical components of lavender, have been suggested to be responsible for reducing anxiety [8].

Recently, some studies reported the effects of nighttime aromatherapy on sleep quality. Exposure to lavender aroma during sleep improved subjective sleepiness in young healthy adults [9]. In another study, Inhaling the aroma of an essential oil during sleep improved sleep quality in patients undergoing cardiac rehabilitation [10]. However, the effects of nighttime aromatherapy on mood states remain unclear. The aim of this study was to determine whether nighttime aromatherapy using skin patches with lavender aroma improves mood states in young women.

\section{Methods}

\section{Subjects}

Subjects were recruited via publicity posters placed in a university campus. The inclusion criterion was healthy female aged over 18 years. The exclusion criteria included pregnant women, lactating mothers, smokers, women who work night shift duty, women who use hypnotics, women who are allergic to lavender, and women with a past history of adverse events associated with aromatherapy.

\section{Study design and procedure}

This study was a randomized, controlled, single-blind trial. The study procedure is illustrated in Fig. 1. Twenty-four subjects were randomly allocated to either an aromatherapy (AROM, $n=12)$ or a control (CONT, $n=12$ ) group. Subjects were instructed to place a skin patch under their clothing and sleep as usual at home, for seven consecutive nights. The AROM group used commercial skin patches with lavender aroma (Aroma patch ${ }^{\circledR}$, Japan Detox, Toyama, Japan), and the CONT group used skin patches without the aroma (EM one-touch pad®, New Tac Kasei Co., Ltd, Kagawa, Japan). The patch was removed and discarded once the subject was awake. Assessment of mood states and the collection of saliva were conducted between 8:30 am and 10:00 am pre-intervention (day 0), the morning after the first intervention night (day 1), and post-intervention (day 7). Pulse rate was measured by the subjects upon waking up before, during, and after the intervention. Subjects were asked to refrain from other aromatherapies during their participation in the study.

\section{Outcome measures}

The primary outcome measure was change in mood states. Secondary outcome measures were physiological indices of stress.

\section{Assessment of mood states}


Mood states were assessed using the Profile of Mood States 2nd Edition (POMS 2). The POMS 2, which is a modified version of the POMS, simultaneously evaluates seven moods: anger-hostility (AH), confusion-bewilderment (CB), depression-dejection (DD), fatigue-inertia (FI), tension-anxiety (TA), vigoractivity (VA), and friendliness (F). The total mood disturbance (TMD) score was calculated using the following formula:

TMD score $=(A H+C B+D D+F I+T A)-V A$

We used the Japanese version of short form POMS 2, which includes 35 questions, to reduce the burden of the assessment on the subjects.

\section{Collection of saliva}

Saliva was collected using a cotton swab (Salivette ${ }^{\circledR}$, SARSTEDT AG \& CO, Numbrecht, Germany). The subjects were not allowed to take alcoholic beverages the night before saliva collection. Eating, drinking (except drinking of water), exercising, and bathing on the morning of the saliva collection were also prohibited. After rinsing the mouth thoroughly, the subjects were instructed to gently chew the swab for 1 minute to allow the swab to absorb saliva. The saliva-saturated swab was placed in a tube and centrifuged at 3,000 rpm for $5 \mathrm{~min}$. Obtained saliva samples were frozen at $-80^{\circ} \mathrm{C}$ until the time of analysis.

\section{Measurement of salivary a-amylase activity and cortisol concentration}

Salivary a-amylase activity was measured by using a colorimetric assay kit (Salivary a-amylase kinetic enzyme assay kit, Salimetrics, LLC, PA, USA). Salivary cortisol concentration was measured via the ELISA method using a salivary cortisol enzyme assay kit (Salimetrics, LLC).

\section{Measurement of pulse rate}

Pulse rate was measured by the subjects, who were instructed to measure the pulse rate of the radial artery immediately after waking up.

\section{Sleep quality and quantity}

Pre-intervention sleep quality was assessed using the Pittsburgh Sleep Quality Index [11] for Japanese (PSQI-J) $[12,13]$. Sleep disorder was considered to be present when an individual's score was $\geq 6[11,12]$. The average amount of sleep (hours) during the intervention (day 0 to day 7) was assessed using the subjects' self-reported data.

\section{Feeling and preference of aroma}

We asked the subjects whether they perceived any aroma on the patches. If they answered, "Yes", we determined their preference of the aroma on a scale of 1 to 5 , with $1=$ I like the aroma very much, $2=$ I like 
the aroma a little, $3=\mathrm{I}$ do not like or dislike the aroma, $4=\mathrm{I}$ hardly like the aroma, and $5=\mathrm{I}$ do not like the aroma.

\section{Statistical analysis}

Data are presented as mean \pm standard error. Data on the subjects' characteristics were analyzed using the unpaired t-test or chi-square test. POMS 2 scores, pulse rates, and salivary biomarkers were analyzed using a two (condition [with/without aromatherapy]) $\times$ three (time of measurement [pre-intervention, the morning after the first intervention night, and post-intervention]) analysis of variance (ANOVA) to determine interaction and main effects. When statistical significance was found by ANOVA, the Bonferroni post-hoc test was used to compare the data. Statistical analyses were performed using SPSS Statistics ver. 25.0 (IBM, Tokyo, Japan). Two-tailed-p-values of $<0.05$ were considered indicative of statistical significance.

\section{Results}

\section{Subject characteristics}

Subject characteristics are shown in Table 1. There were no significant differences in characteristics between the groups. More than $50 \%$ of the subjects in both groups were diagnosed with a sleep disorder. The incidence of sleep disorder in the present study is similar to that in a previous study that demonstrated sleep problems in Japanese university students [14]. 
Table 1

Subject characteristics

\begin{tabular}{|llll|}
\hline & $\begin{array}{l}\text { AROM } \\
(\mathbf{n = 1 2})\end{array}$ & $\begin{array}{l}\text { CONT } \\
(\mathbf{n = 1 2})\end{array}$ & p-value \\
\hline Age (years) & $21.0 \pm 0.4$ & $20.4 \pm 0.4$ & 0.278 \\
\hline Body height $(\mathrm{cm})$ & $160.8 \pm 1.9$ & $160.4 \pm 2.0$ & 0.895 \\
\hline Body weight $(\mathrm{kg})$ & $55.2 \pm 2.0$ & $53.3 \pm 2.0$ & 0.515 \\
\hline BMI (kg/m $\left.{ }^{2}\right)$ & $21.3 \pm 0.5$ & $20.7 \pm 0.5$ & 0.385 \\
\hline PSQI-J (score) & $5.8 \pm 0.9$ & $5.7 \pm 0.6$ & 0.881 \\
\hline Sleep disorder (n) & $7(58.3 \%)$ & $6(50.0 \%)$ & 0.682 \\
\hline Sleeping hour (hours) & $6.7 \pm 0.2$ & $6.5 \pm 0.2$ & 0.608 \\
\hline $\begin{array}{l}\text { AROM, aromatherapy group; BMI, body mass index; CONT, control group; PSQI-J, the Pittsburgh Sleep } \\
\text { Quality Index for Japanese }\end{array}$ & & \\
\hline $\begin{array}{l}\text { Values, except sleep disorder, are expressed as means and SEM. Value of sleep disorder is expressed } \\
\text { as number of subjects (rate). }\end{array}$ & & \\
\hline Sleep disorder is defined as PSQI-J $\geq 5.5$. & & \\
\hline $\begin{array}{l}\text { The average of sleep hours was calculated by self-reported data during intervention period (from day } \\
\text { 0 to day 7). }\end{array}$ & \\
\hline
\end{tabular}

\section{Mood states}

Baseline POMS 2 scores are shown in Table 2. Pre-intervention TMD and POMS 2 subscale scores were not significantly different between the groups. 
Table 2

Baseline POMS 2 score

\begin{tabular}{|c|c|c|c|}
\hline & $\begin{array}{l}\text { AROM } \\
(n=12)\end{array}$ & $\begin{array}{l}\text { CONT } \\
(n=12)\end{array}$ & p-value \\
\hline TMD & $45.8 \pm 1.7$ & $45.6 \pm 2.4$ & 0.956 \\
\hline $\mathrm{AH}$ & $43.1 \pm 2.1$ & $43.8 \pm 2.4$ & 0.814 \\
\hline CB & $46.3 \pm 2.0$ & $48.5 \pm 3.2$ & 0.570 \\
\hline DD & $46.6 \pm 2.3$ & $46.6 \pm 1.7$ & 1.000 \\
\hline $\mathrm{FI}$ & $49.2 \pm 1.7$ & $45.3 \pm 2.3$ & 0.184 \\
\hline TA & $44.7 \pm 2.3$ & $45.3 \pm 3.3$ & 0.887 \\
\hline VA & $50.0 \pm 2.8$ & $47.0 \pm 2.2$ & 0.414 \\
\hline $\mathrm{F}$ & $53.0 \pm 22.2$ & $49.8 \pm 2.8$ & 0.384 \\
\hline \multicolumn{4}{|c|}{$\begin{array}{l}\text { AH, anger-hostility; AROM, aromatherapy group; } \mathrm{CB} \text {, confusion-bewilderment; CONT, control group; DD } \\
\text { depression-dejection; F, friendliness; Fl, fatigue-inertia; POMS 2, the Profile of Mood States } 2 \text { nd Edition } \\
\text { TA, tension-anxiety; TMD, total mood disturbance; VA, vigor-activity }\end{array}$} \\
\hline \multicolumn{4}{|c|}{ Values are expressed as means and SEM. } \\
\hline
\end{tabular}

Figure 2A shows chronological changes in the TMD. Regarding the TMD score, two-way ANOVA showed a statistical difference in TME scores between time $(p<0.05)$, but no interaction was detected between time and condition $(p=0.800)$. After Bonferroni's test, the TMD scores on days 1 and 7 were significantly lower than those on day 0 in the AROM group, but no significant difference in TMD scores was observed between time in the CONT group.

Figures 2B-2F show chronological changes in the negative subscale scores of the POMS 2. Concerning the FI score, two-way ANOVA showed an interaction between time and condition $(p<0.05)$ and a statistical difference in time $(p<0.01)$. After Bonferroni's test, the FI score on day 7 was significantly lower than that on day 0 in the AROM group, but there was no significant difference in FI scores between time in the CONT group (Fig. 2E). Regarding the AH score, two-way ANOVA showed a statistical difference in time $(p<0.05)$, but no interaction was detected between time and condition $(p=0.341)$. After Bonferroni's test, the AH score on day 7 was significantly lower than that on day 0 in the AROM group, but there was no significant difference in AH scores between time in the CONT group (Fig. 2B). For the $\mathrm{CB}$, DD, and TA scores, two-way ANOVA showed no significant differences in time (CB: $p=0.064$; DD: $p=$ 0.119; TA: $p=0.111$ ), and no interaction was noted between time and condition (CB: $p=0.540 ; \mathrm{DD}: p=$ 0.663 ; TA: $p=0.521$ ) (Figs. 2C, 2D, and 2F).

Figures $2 \mathrm{G}$ and $2 \mathrm{H}$ show chronological changes in the positive subscale scores of the POMS 2 . For the VA and F scores, two-way ANOVA showed no significant difference in time (VA: $p=0.250 ; \mathrm{F}: p=0.515$ ), 
and no interaction was observed between time and condition (VA: $p=0.604 ; \mathrm{F}: p=0.662$ ).

\section{Salivary a-amylase activity, cortisol concentration, and pulse rate}

Figures $3 \mathrm{~A}$ and $3 \mathrm{~B}$ show chronological changes in salivary a-amylase activity and cortisol concentration. Salivary a-amylase activity and cortisol concentration pre-intervention were not significantly different between the groups (a-amylase: AROM, $50.3 \pm 16.5 \mathrm{U} / \mathrm{mL}$; CONT, $58.0 \pm 10.9 \mathrm{U} / \mathrm{mL}, p=0.701$; cortisol: AROM, $0.92 \pm 0.15 \mu \mathrm{g} / \mathrm{dL} ; C O N T, 1.23 \pm 0.14 \mu \mathrm{g} / \mathrm{dL}, p=0.145)$. For salivary a-amylase activity, two-way ANOVA showed a statistical difference in time $(p<0.05)$, but no interaction was detected between time and condition $(p=0.532)$. After Bonferroni's test, salivary a-amylase activity on day 1 was significantly higher than that on day 0 in the AROM group, but there were no significant differences in salivary aamylase activity between pre- and post-intervention (Fig. 3A). There were no significant differences in salivary a-amylase activity between time in the CONT group (Fig. 3A). For salivary cortisol concentration, two-way ANOVA showed no significant differences in time $(p=0.162)$, and no interaction was found between time and condition ( $p=0.669)$ (Fig. 3B).

Figure 4 shows chronological changes in pulse rate from day 0 to day 7 . Pre-intervention pulse rate was not significantly different between the groups (AROM: $63.5 \pm 2.1 \mathrm{bpm}$, CONT: $62.3 \pm 3.3 \mathrm{bpm}, p=0.752$ ). Further, two-way ANOVA showed no significant difference in pulse rate between time $(p=0.141)$ and between condition $(p=0.550)$.

\section{Feeling and preference for aroma}

All subjects in the AROM group responded that they perceived the lavender aroma. In addition, all participants in the CONT group responded that they did not perceive the lavender aroma.

Regarding preference for the aroma, 1, 2, 3, 4, and 5 subjects answered with 1, 3, 4, 3 and 1, respectively, on a scale of 1 to 5 .

\section{Adverse effects}

No adverse effects were observed during the study period.

\section{Discussion}

The use of skin patches with lavender aroma during sleep improved TMD scores and the scores of two negative subscales of POMS 2 ( $\mathrm{AH}$ and $\mathrm{FI}$ ) in the young women over time. For $\mathrm{Fl}$, an interaction was detected between time and condition, and FI was significantly reduced compared to baseline, after 7 days of nighttime aromatherapy. This result suggests that using skin patches with the lavender aroma during sleep significantly improved FI. However, no interactions between time and condition were detected for TMD and $\mathrm{AH}$, although these scores were significantly reduced, compared to baseline, after 7 days of nighttime aromatherapy. These results suggest that the effect of nighttime aromatherapy on mood states 
did not sufficiently exceed the placebo effect. A similar placebo effect on acute stress was shown in young healthy adults receiving aromatherapy [15]. In addition, the effect of lavender aromatherapy on psychological indices of relaxation were considered to have been related to the subjects' expectation, not to the aroma, in young healthy women [16]. The results of present study, which was single-blind, may have been associated with expectancy biases, although all participants in the CONT group mentioned that they did not perceive the lavender aroma.

The baseline states of the participants may have also affected the results of the present study. Olfactory function and odor performance is influenced by age, sex, clinical state, current medication, season of the year, and time of the day [17-19]. In a meta-analysis, anxiolytic effects of aromatherapy were observed mainly in patients with anxiety [3]. In healthy subjects, the aroma of lavender reduced mental stress [15, 20]. The implementation of overnight aromatherapy using lavender oil patches, similar to the present study, improved sleep quality and duration and also reduced anxiety in inpatients in a hematologyoncology unit [21]. The POMS 2 scores of the majority of the participants in the present study were within the normal range. Therefore, the effects of the lavender aroma may have not been appreciable in the present study.

Only a few studies have examined the effects of aroma on biomarkers for stress [22]. In the present study, salivary a-amylase level and cortisol concentration were measured as indices of stress response via the hypothalamic-sympathetic nervous system and hypothalamic-pituitary-adrenal (HPA) axes. Salivary aamylase level measured on the morning after the night of the first intervention was significantly higher than that at baseline. This result suggests that sympathetic nervous activity, at least in the morning, was increased by the use of skin patches with a lavender aroma. However, no interaction was found between time and condition for salivary a-amylase level. Therefore, our results do not indicate that lavender aroma increases sympathetic nervous activity. Furthermore, a previous study showed that salivary a-amylase level was decreased by the inhalation of lavender aroma during the inhalation period, but salivary aamylase level returned to the baseline level during the post-inhalation period [23]. Alpha-amylase level is considered to reflect hyperacute psychosomatic reactivity to aroma. Therefore, salivary a-amylase may have not been able to detect the chronic effect of aroma on stress in the present study.

There were no significant differences in salivary cortisol concentration between the groups in the present study. A few studies which examined the acute effect of lavender aroma inhalation reported that salivary cortisol level did not change in the healthy young adults [23, 24]; however, no study has reported the chronic effect of lavender aroma inhalation on salivary cortisol level. The results of the present study suggest that lavender aroma may not affect stress response via the HPA axis. In addition, the baseline states of the participants may have affected the physiological indices. Further studies are needed to demonstrate the beneficial effects of nighttime aromatherapy on physiological stress responses in subjects with high stress.

There were also no significant differences in pulse rate caused between participants who used or did not use the skin patches in the present study. Heart rate is modulated by cardiac autonomic nervous activity, 
and reduction in heart rate reflects a reduction of sympathetic nervous activity and/or the enhancement of parasympathetic nervous activity. Lavender, especially its linalool component, affects the autonomic nervous system, inhibits sympathetic nervous activity, and excites parasympathetic nervous activity [4]. Lavender aromatherapy was shown to decrease heart rate in patients with cardiovascular disease [2527]. However, in healthy individuals, changes in heart rate caused by the inhalation of the lavender aroma were inconsistent $[20,28]$. Thus, the effect of the lavender aroma on heart rate may be more profound in patients with disorders of the autonomic nervous system than in the healthy adults.

There are many methods and essential oils for aromatherapy [1]. Very recently, a systematic review and network meta-analysis regarding the best route of administration of lavender was conducted [29]. However, few studies have used skin patches for aromatherapy. Aromatherapy using skin patches is advantageous because it is easily applicable, non-invasive, safe, and inexpensive. However, the optimal treatment method, such as the aroma type and dose, remain unclear.

The present study had a few limitations. First, the sample size was small. Second, the participants were young, healthy women. These factors may limit the generalizability of the findings of the present study. Despite these limitations, the results of the present study suggest that aromatherapy during sleep may be useful for stabilizing mood and states. Further studies are needed to demonstrate the beneficial effects of nighttime aromatherapy on mood states in various populations.

\section{Conclusion}

The use of skin patches with lavender aroma during sleep partly improved mood states in young women. The result of the present study indicates that nighttime aromatherapy using skin patches with lavender aroma is an easy, inexpensive, and safe approach that can stabilize the mood states of an individual.

\section{Abbreviations}

$\mathrm{AH}$ : anger-hostility

ANOVA: analysis of variance

AROM: aromatherapy

BMI: body mass index

CB: confusion-bewilderment

CONT: control

DD: depression-dejection

$F$ : friendliness 
FI: fatigue-inertia

PSQI-J: the Pittsburgh Sleep Quality Index for Japanese

POMS 2: the Profile of Mood States 2nd Edition

SEM: standard error

TA, tension-anxiety

TMD: total mood disturbance

VA: vigor-activity

\section{Declarations}

\section{Ethics approval and consent to participate}

The ethical committee of Teikyo University approved this study (Approval number: 18-050), and the experiment was performed in conformity with the standards set by the Declaration of Helsinki. Subjects provided written informed consent to participate in this study.

\section{Consent for publication}

Not applicable.

\section{Availability of data and materials}

The datasets used and/or analysed during the current study are available from the corresponding author on reasonable request.

\section{Competing interests}

The authors declare that they have no competing interests.

\section{Funding}

Financial support was provided by the Teikyo University. This study did not receive funding from external funding sources.

\section{Author's contributions}

KS and YO conceived and designed the experiments. KS, YO, and SK performed the experiments. KS and YO analyzed the data. SK contributed reagents/analysis tools. KS and YO prepared a draft of the manuscript. SK edited the manuscript. The corresponding author and all coauthors reviewed and approved the manuscript. 


\section{Acknowledgments}

The authors acknowledge the subjects who voluntarily participated in this study. The authors would like to thank Editage (https://www.editage.jp/) for providing English language review.

\section{Authors' information}

Affiliations

Department of Sport and Medical Science, Faculty of Medical Technology, Teikyo University, 359 Otsuka, Hachioji, Tokyo 192-0395, Japan

Kanako Sazawa, Yoshiko Ogawa \& Shigeo Kawada

Corresponding author

Correspondence to Yoshiko Ogawa.

\section{References}

1. Freeman M, Ayers C, Peterson C, Kansagara D. Aromatherapy and Essential Oils: A Map of the Evidence. Washington (DC): Department of Veterans Affairs (US), 2019.

2. PDQ Integrative, Alternative, and Complementary Therapies Editorial Board. Aromatherapy With Essential Oils $(\mathrm{PDQ} \circledast)$ : Health Professional Version. PDQ Cancer Information Summaries [Internet]. Bethesda (MD): National Cancer Institute (US); 2002-2019.

3. Kang HJ, Nam ES, Lee Y, Kim M. How Strong is the Evidence for the Anxiolytic Efficacy of Lavender?: Systematic Review and Meta-analysis of Randomized Controlled Trials. Asian Nurs Res 2019;13:295305.

4. Koulivand PH, Khaleghi Ghadiri M, Gorji A. Lavender and the nervous system. Evid Based Complement Alternat Med 2013;2013:681304.

5. Diego MA, Jones NA, Field T, Hernandez-Reif M, Schanberg S, Kuhn C, et al. Aromatherapy positively affects mood, EEG patterns of alertness and math computations. Int J Neurosci 1998;96:217-24.

6. Hwang E, Shin S. The effects of aromatherapy on sleep improvement: a systematic literature review and meta-analysis. J Altern Complement Med. 2015;21:61-8.

7. Lillehei AS, Halcon LL. A systematic review of the effect of inhaled essential oils on sleep. J Altern Complement Med. 2014 Jun;20:441-51.

8. Setzer WN. Essential oils and anxiolytic aromatherapy. Nat Prod Commun. 2009;4:1305-16.

9. Hirokawa K, Nishimoto T, Taniguchi T. Effects of lavender aroma on sleep quality in healthy Japanese students. Percept Mot Skills. 2012;114:111-22.

10. McDonnell B, Newcomb P. Trial of Essential Oils to Improve Sleep for Patients in Cardiac Rehabilitation. J Altern Complement Med. 2019;25:1193-9. 
11. Buysse DJ, Reynolds CE, Monk TH, Berman SR, Kupfer DJ. The Pittsburgh Sleep Quality Index: a new instrument for psychiatric practice and research. Psychiatry Res 1989; 28:193-213.

12. Doi Y, Minowa M, Uchiyama M, Okawa M, Kim K, Shibui K, et al. Psychometric assessment of subjective sleep quality using the Japanese version of the Pittsburgh Sleep Quality Index (PSQI-J) in psychiatric disordered and control subjects. Psychiatry Res 2000;97:165-72.

13. Doi Y, Minowa M, Okawa M, Uchiyama M. Development of the Japanese version of the Pittsburgh Sleep Quality Index. Japanese Journal of Psychiatry Treatment 1998;13:755-63 (in Japanese).

14. Kayaba M, Matsushita T, Enomoto M, Kanai C, Katayama N, Inoue Y, et al. Impact of sleep problems on daytime function in school life: a cross-sectional study involving Japanese university students. BMC Public Health 2020;20:371.

15. Sgoutas-Emch S, Fox T, Preston M, Brooks C, Serber E. Stress management: aromatherapy as an alternative. Sci Rev Altern Med 2001;5:90-5.

16. Howard S, Hughes BM. Expectancies, not aroma, explain impact of lavender aromatherapy on psychophysiological indices of relaxation in young healthy women. Br J Health Psychol 2008;13(Pt 4):603-17.

17. Goel N, Grasso DJ. Olfactory discrimination and transient mood change in young men and women: variation by season, mood state, and time of day. Chronobiol Int 2004;21:691-719.

18. Brand G, Millot JL. Sex differences in human olfaction: between evidence and enigma. Q J Exp Psychol B 2001;54:259-70.

19. Ship JA, Weiffenbach JM. Age, gender, medical treatment, and medication effects on smell identification. J Gerontol 1993;48:M26-32.

20. Motomura N, Sakurai A, Yotsuya Y. Reduction of mental stress with lavender odorant. Percept Mot Skills $2001 ; 93: 713-8$.

21. Shady K, Nair JM, Crannell C. Lavender Aromatherapy: Examining the Effects of Lavender Oil Patches on Patients in the Hematology-Oncology Setting. Clin J Oncol Nurs. 2019;23:502-8.

22. Hur MH, Song JA, Lee J, Lee MS. Aromatherapy for stress reduction in healthy adults: a systematic review and meta-analysis of randomized clinical trials. Maturitas 2014;79:362-9.

23. Yamaguchi M, Tahara Y, Kosaka S. Influence of concentration of fragrances on salivary alphaamylase. Int J Cosmet Sci. 2009;31:391-5.

24. Toda M, Morimoto K. Effect of lavender aroma on salivary endocrinological stress markers. Arch Oral Biol. 2008;53:964-8.

25. Bahrami T, Rejeh N, Heravi-Karimooi M, Vaismoradi M, Tadrisi SD, Sieloff C. Effect of aromatherapy massage on anxiety, depression, and physiologic parameters in older patients with the acute coronary syndrome: a randomized clinical trial. Int J Nurs Pract 2017;23:e12601.

26. Ziyaeifard M, Azarfarin R, Faritous Z, Dehdashtia, E, Baghestani A, Ziyaeifard P, et al. Evaluation of lavender oil inhalation effects on blood pressure and heart rate in patients undergoing coronary angiography. Iran Heart J 2017;18:29-33. 
27. Rajai N, Sajadi SA, Teymouri F, Zareiyan A, Siavoshi S, Malmir M. The effect of aromatherapy with lavender essential oil on anxiety and stress in patients undergoing coronary artery bypass graft surgery. Jundishapur J Chronic Dis Care 2016;5:e34035.

28. Sayorwan W, Siripornpanich V, Piriyapunyaporn T, Hongratanaworakit T, Kotchabhakdi N, Ruangrungsi $\mathrm{N}$. The effects of lavender oil inhalation on emotional states, autonomic nervous system, and brain electrical activity. J Med Assoc Thai 2012;95:598-606.

29. Sayed AM, Morsy S, Tawfik GM, Naveed S, Minh-Duc MT, Hieu TH, et al. The best route of administration of lavender for anxiety: a systematic review and network meta-analysis. Gen Hosp Psychiatry. 2020;64:33-40.

\section{Figures}

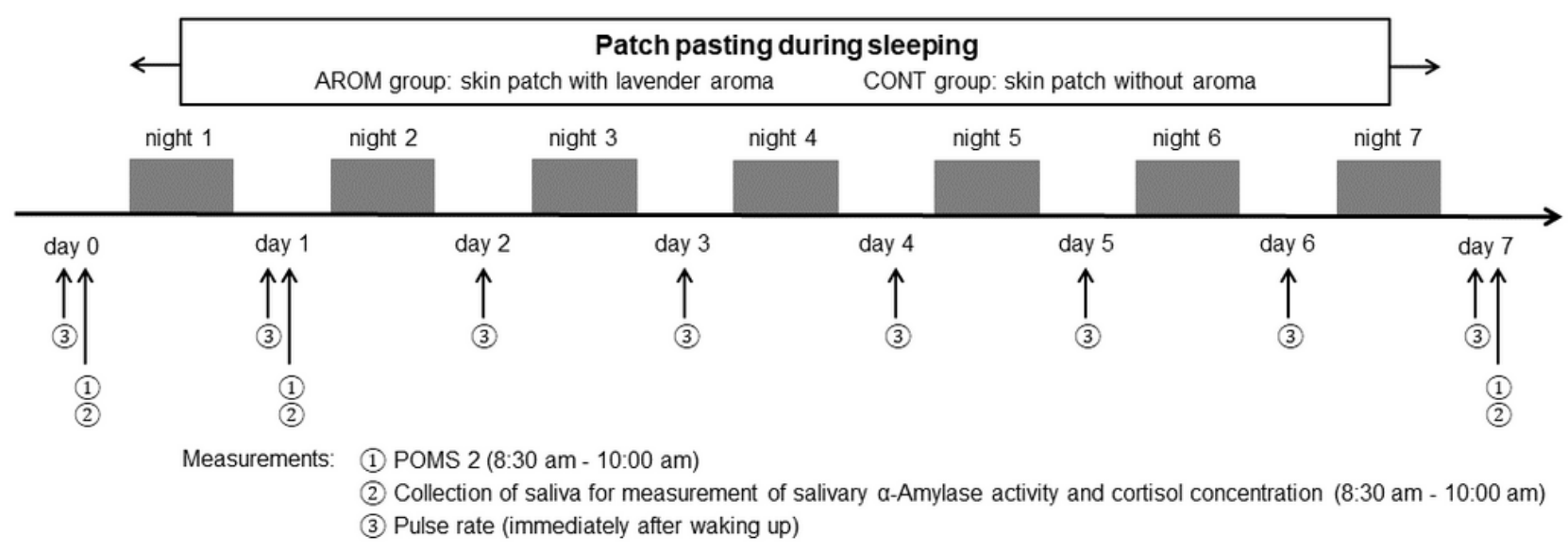

\section{Figure 1}

AROM, aromatherapy; CONT, control; POMS 2, the Profile of Mood States 2nd Edition 

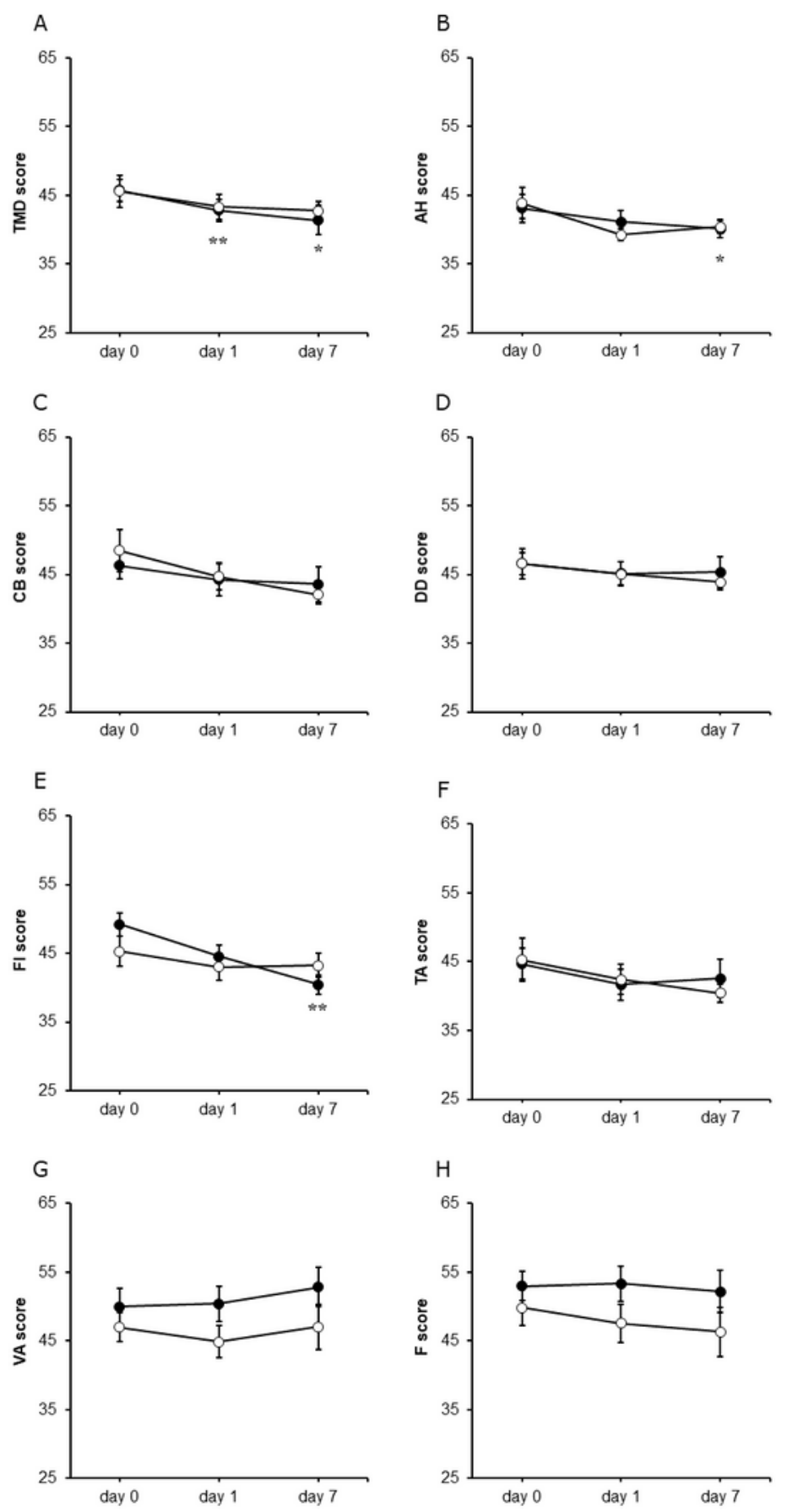

\section{Figure 2}

Chronological changes in the POMS 2 score Chronological changes in the TMD (A), AH (B), CB (C), DD (D), FI (E), TA (F), VA (G), F (H) in the AROM $(\bullet, n=12)$ and CONT $(\bullet, n=12)$ groups. AH, anger-hostility; AROM, aromatherapy; $C B$, confusion-bewilderment; CONT, control; DD, depression-dejection; $F$, friendliness; Fl, fatigue-inertia; POMS 2, the Profile of Mood States 2nd Edition; TA, tension-anxiety; TMD, 
total mood disturbance; VA, vigor-activity Data are expressed as means $\pm S E M .{ }^{*} p<0.05,{ }^{\star *} p<0.01$ compared with the score on day 0 in the AROM group.
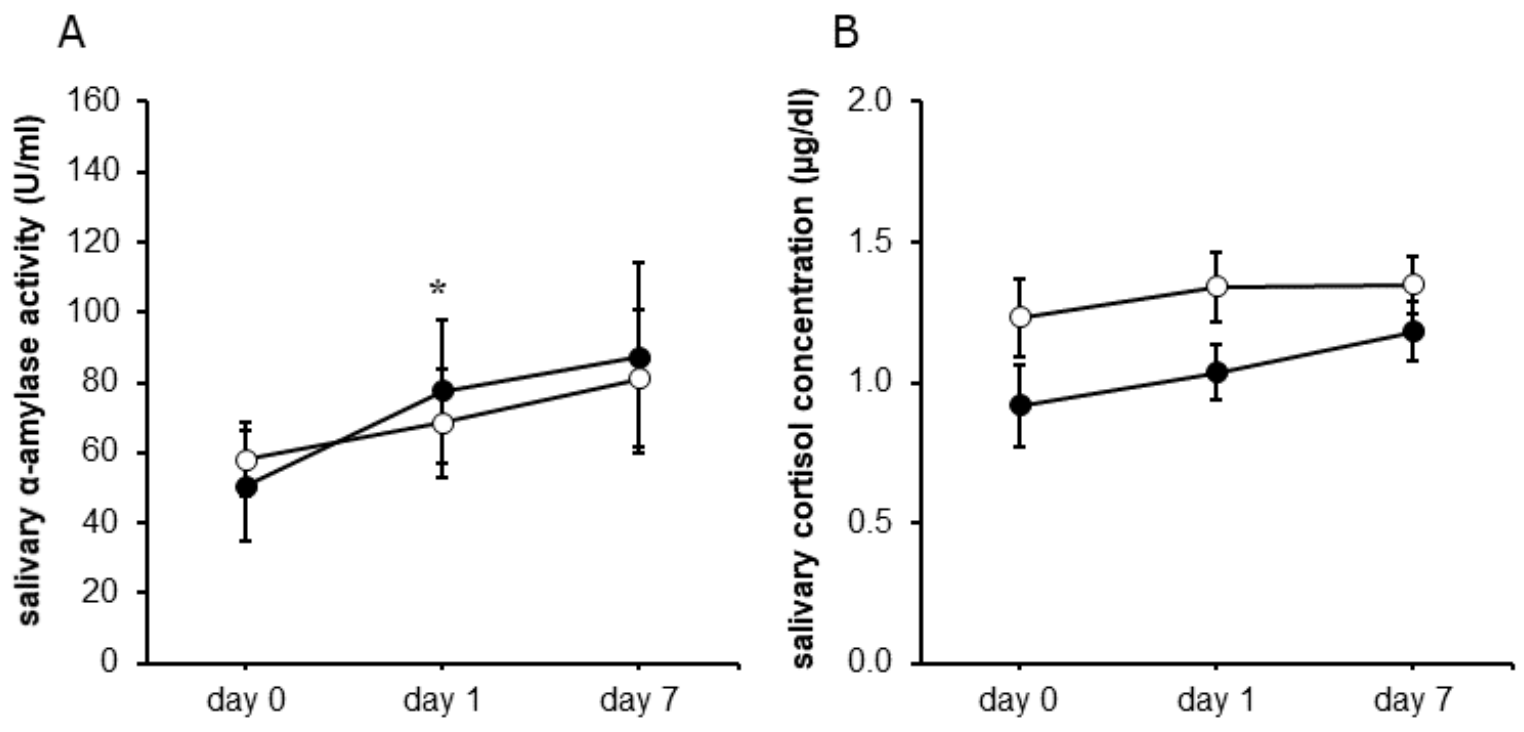

\section{Figure 3}

Chronological changes in salivary a-amylase activity and cortisol concentration Chronological changes in salivary a-amylase activity $(A)$ and cortisol concentration $(B)$ in the $\operatorname{AROM}(\boldsymbol{O}, n=12)$ and CONT $(\mathbf{0}, \mathrm{n}=$ 12) groups. AROM, aromatherapy; CONT, control Data are expressed as means $\pm S E M . * p<0.05$ compared with the score on day 0 in the AROM group. 


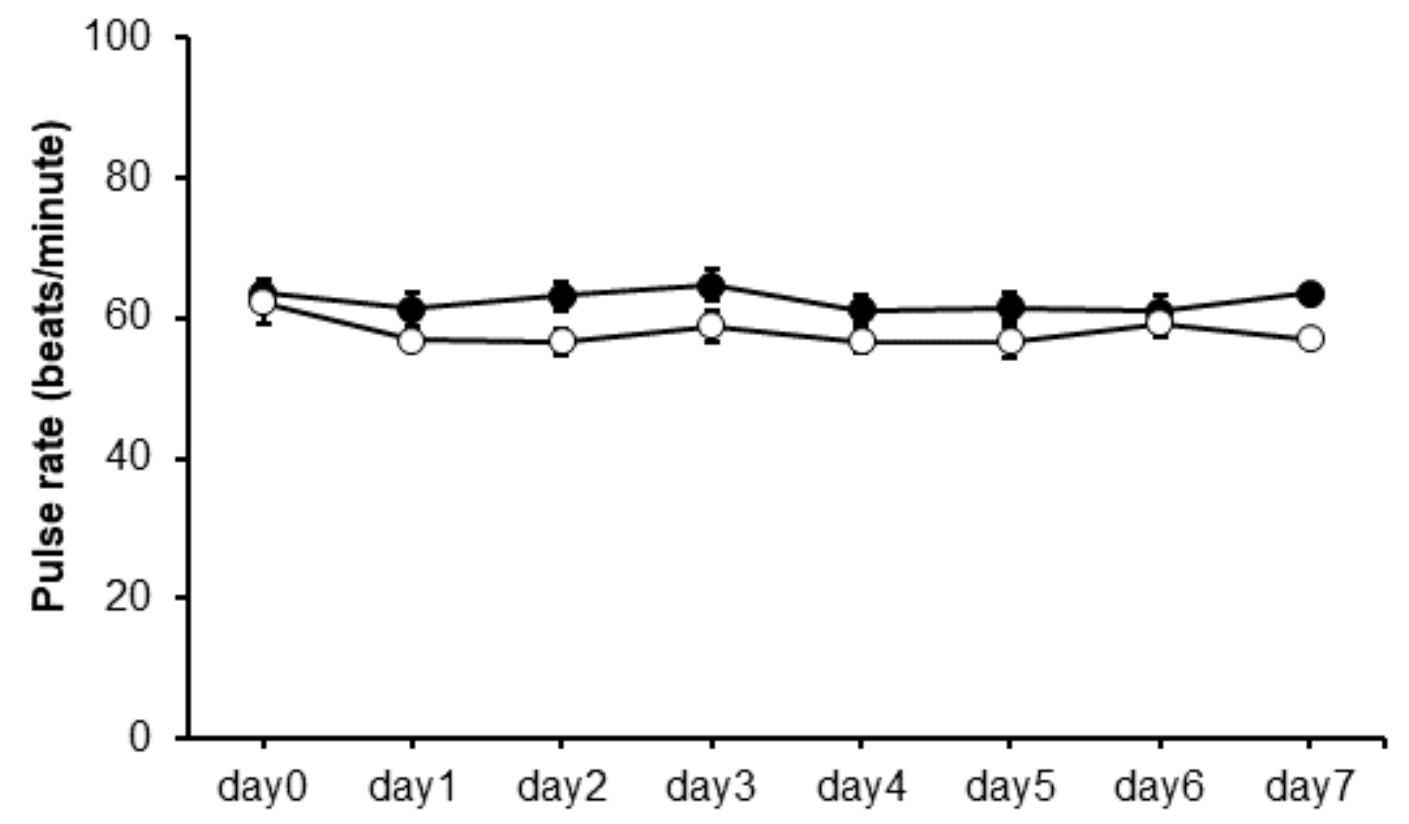

Figure 4

Chronological change in pulse rate Chronological changes in pulse rate based on subject-measured data. Measurements were performed from day 0 to day 7, immediately after the individual woke up in the $\operatorname{AROM}(\bullet, n=12)$ and CONT $(\bullet, n=12)$ groups. AROM, aromatherapy; CONT, control Data are expressed as means \pm SEM. 